\title{
THE BJERKNES' CIRCULATION THEOREM A Historical Perspective
}

\author{
by Alan J. Thorpe, Hans Volkert, and Michat J. Ziemiański
}

Other physicists had already made the mathematical extension of Kelvin's theorem to compressible fluids, but it was not until Vilhelm Bjerknes' landmark 1898 paper that meteorology and oceanography began to adopt this insight.

$\mathrm{T}$ his essay is primarily about the Bjerknes circulation theorem (Bjerknes 1898). This theorem, formulated by Vilhelm Bjerknes, was published in a paper of 1898, in the Proceedings of the Royal Swedish Academy of Sciences in Stockholm. The paper is in German, being one of the primary languages of scientific publications of that era. The title of the paper is "On a Fundamental Theorem of Hydrodynamics and Its Applications Particularly to the Mechanics of the Atmosphere and the World's Oceans." It has 35 pages, 36 sections, and 14 figures. The significance of this paper is twofold. It provided a key

AFFILIATIONS: THORPE-NERC Centres for Atmospheric Science, University of Reading, Reading, United Kingdom; VOLKERT-Institut für Physik der Atmosphäre, DLR Oberpfaffenhofen,

Oberpfaffenhofen, Germany; ZIEMIAŃSKI-Institute of Meteorology and Water Management, Gdynia, Poland

CORRESPONDING AUTHOR: Prof. Alan Thorpe, Dept. of Meteorology, University of Reading, Earley Gate, P.O. Box 243, Reading RG6 6BB, United Kingdom

E-mail: a.j.thorpe@reading.ac.uk

DOI: 10.1 175/BAMS-84-4-47।

(C2003 American Meteorological Society

insight into the way circulation develops in geophysical fluids. But it also marked the beginning of the transition of Vilhelm Bjerknes' research from the field of electrostatics, electromagnetic theory, and pure hydrodynamics into that of atmospheric physics.

Prior to the introduction of this theorem the thinking on rotation in a fluid followed two distinct lines. The quantity we now call vorticity (curl of the velocity vector) had been the subject of fundamental studies by $\mathrm{H}$. Helmholtz, although its recognition as a fluid property predates these studies by at least 80 years. In Helmholtz (1858) equations for the rate of change of vorticity had been derived for a homogeneous inviscid fluid. Helmholtz thereby deduced that for a constant density fluid a material line element (a "vortex filament") aligned with the vorticity vector will always remain so aligned. In essence if no vorticity currently exists, then none could be generated by conservative forces.

Lord Kelvin, in a paper in 1867 (Thomson 1867), had approached the problem of rotation in a different way by defining a quantity called circulation given by the following expression:

$$
C=\oint \mathbf{u} \cdot d \mathbf{l}
$$


where the integral is around any closed curve in the fluid and $d \mathbf{l}$ is a line element vector pointing along the curve. Kelvin's theorem states that the circulation around a material circuit is constant for a homogeneous inviscid fluid. A material circuit is one that always consists of the same fluid parcels.

By using Stokes's theorem one can easily derive the relationship between the relative circulation, $C$, and the relative vorticity:

$$
C=\iint \zeta \cdot d \mathbf{A},
$$

where the integrals are over any surface with a perimeter defined by the aforementioned closed curve, $\zeta$ is the relative vorticity vector, and $d \mathbf{A}$ is an elemental area vector pointing normal to the surface. In other words, circulation is an area average of the normal component of vorticity. Vorticity is a differentiated quantity, mathematically defined at a point, and so it is not easy to observe at small scales. Therefore an area average, that is, the circulation, is all that actual observations can define.

Both Helmholtz's vorticity equation and Kelvin's circulation theorem have limited applicability to the atmosphere because they refer to a constant density fluid. In 1895 J. R. Schütz (Schütz 1895), a German physicist working at the University of Göttingen, extended Helmholtz's vorticity equations to the case of a compressible fluid. In a short note he showed that vorticity can be generated by terms in the equation depending on spatial gradients of density and pressure. For example,

$$
\frac{D \zeta}{D t}=\frac{\partial}{\partial x}\left(\frac{1}{2 \rho} \frac{\partial p}{\partial y}\right)-\frac{\partial}{\partial y}\left(\frac{1}{2 \rho} \frac{\partial p}{\partial x}\right),
$$

where $\zeta$ is the vertical component of the vorticity vector and it is interesting to note that at that time it was common practice to define vorticity as one-half of the curl of the velocity vector. While it is clear that the right-hand side of (3) can be written as the cross product of the density and pressure gradients, Schütz did not comment on this. He looks for conditions where the right-hand side of (3) vanishes, that is, vorticity is conserved, and states that this is the case when $d p / \rho$ is a "total differential." This presages the importance of this quantity in the circulation theorem. In a footnote, Schütz notes that "for one who likes a paradox they may say that vortex motions are impossible in incompressible fluids, but easily generated in fluids with indefinitely small compressibility."
In 1896 Ludwik Silberstein, a Polish physicist born in Warsaw, used Schütz's extension of Helmholtz's vorticity equations to consider the cause of the emergence of rotation in a nonhomogeneous fluid that initially has no such rotation. He considered a gas in which pressure and density surfaces may not coincide, which of course is the case in the atmosphere. Silberstein's paper appeared as a brochure published in Polish by the Academy of Sciences in Cracow [Silberstein (1896), and the following year, 1897, in the Proceedings of the Cracow Academy of Sciences] with the title "About the Creation of Eddies in the Ideal Fluid." These papers were published when Silberstein was 24 years old, working as a research assistant in the physics laboratory of the Polytechnic in Lvov (about $300 \mathrm{~km}$ east of Cracow). Silberstein was interested, prior to 1909, in electromagnetic theory and the theory of electrons. For the remainder of his career (he died in 1948) he was concerned with the theory of relativity. His name is associated with the quaternion form of the special theory of relativity and in 1912 he wrote a paper on this in Philosophical Magazine (Średniawa 1997).

Silberstein (1896) asked the question about what distribution of density and pressure must occur in an ideal (frictionless) fluid to create vorticity if none was currently in existence, hence the title referring to the creation of eddies. Studying a general threedimensional flow he showed that the problem of vorticity creation can be understood, from a physical viewpoint, in terms of intersecting surfaces of constant pressure and density. This arises directly from his realization that the right-hand side of (3), for example, can be written as the Jacobian of the pressure and density fields. In particular Silberstein shows that the orientation of developing vortex filaments is the same as that of the intersection line of constant pressure and density surfaces. Furthermore he discusses that the intersection of these surfaces is the necessary and sufficient condition for the generation ("acceleration") of vorticity in a fluid. Finally he shows that the vorticity generation depends on the cross product of the gradient of pressure and that of density. Silberstein's second theorem (Silberstein 1896) states that

The elements of a frictionless fluid lacking vortical motion do not start to rotate if, and only if, the pressure $p$ can be expressed as a function of density $\rho$ only, so that the surfaces of $p$ and $\rho$ coincide with each other....

The relative disposition of the pressure, $p$, and density, $\rho$, surfaces and the sense of the ensuing rotation 
are described in Fig. 1 from Silberstein (1896, p. 329) and are reproduced here as Fig. 1. This results from his derivation, from (3), of the following equation for the rate of change of the magnitude of the vorticity, $\omega$ :

$$
\frac{D}{D t} \omega=\omega^{\prime}=\frac{1}{2 \rho^{2}} \frac{\partial p}{\partial n} \frac{\partial \rho}{\partial v} \sin (v, n),
$$

where $n$ and $v$ are normal to surfaces of constant pressure and density, respectively, pointing toward their increasing values. This result for vortex filaments was also extended for vortex tubes.

In most essential respects Silberstein's paper discovered (first) all the fundamental aspects to be discussed by Bjerknes in his famous paper two years later. Bjerknes was aware of Silberstein's work and references his paper in the 1898 work. The Silberstein (1896) paper was also published, in addition to Polish, in a German translation (without the figures, in a version of the journal with a French name!). Note that Gill (1982) erroneously supposes that Bjerknes was not aware of the earlier Silberstein paper. As we shall see, Bjerknes was clearly the first to show that this vorticity equation (in the circulation form) can be used to explain the properties of geophysical fluids.

\section{VILHELM BJERKNES' CIRCULATION} THEOREM. It is fair to say that Silberstein, similar to Schütz before him, viewed his result as "mathematical corollaries" and felt that "without conducting appropriate scientific research" it was impossible to say how the conditions for creating eddies could actually arise. Bjerknes took exactly the opposite approach focusing in great detail on the practical application of these results.

A few biographical details help set the scene for Bjerknes introduction of his circulation theorem. In 1898, when he was 36, Vilhelm Bjerknes was professor of mathematical physics at the University of Stockholm. Vilhelm was the son of a noted applied mathematician, Carl Anton Bjerknes, who specialized in understanding the electromagnetic field. He pursued hydrodynamic analogies to electromagnetic phenomena in order to establish the existence and properties of the "ether"- the supposed physical medium through which electromagnetic interaction occurred. Vilhelm spent the early part of his scientific career assisting his father. He had studied under Heinrich Hertz who in turn was a student of Helmholtz. The hydrodynamic analogies (and laboratory realizations of these) demanded that Vilhelm develop a deep knowledge of hydrodynamics.


Fig. I. Reproduction of Fig. I from Silberstein (1896). The unit vector $\boldsymbol{n}$ is normal to isobaric surfaces, pointing toward high pressure, and the unit vector $v$ is normal to the density surfaces, pointing toward high density. The symbol $\omega^{\prime}$ stands for the rate of change of the magnitude of the vorticity following a fluid parcel. Silberstein states that, "The axis of the developing eddy collocates with the appropriate element of the intersection line (of constant density and pressure surfaces): the fluid element begins to rotate around this line element from $v$ toward $n$ (along the shorter part) with vortical acceleration $\omega^{\prime}$."

The application of this knowledge to the atmosphere arose from conversations with his friend Nils Ekholm, then an assistant at the Swedish weather service and lecturer in meteorology at the university. Ekholm encouraged Bjerknes to make the transition to geophysical sciences. It was Ekholm who noted by plotting density charts that pressure and density fields were not coincident (as was generally believed at that time) and that cyclones formed in regions of rarified air; see Fig. 32 of Kutzbach (1979), which shows a figure from Ekholm (1891), and which is reproduced here as Fig. 2. Kutzbach, in her excellent book, suggests that Bjerknes "consequently applied the circulation theorem he had developed in 1897 in the course of his studies in hydrodynamics and electromagnetism."

This brings us to the discussion of the Bjerknes (1898) paper. In many ways it is a remarkable paper. It has very few equations or numerical values and so is entirely a discourse on the qualitative geophysical implications of the theorem. The first 14 pages (containing 11 sections) set the scene prior to the mathematical statement of the theorem. On the first page Bjerknes refers to one of his working papers published in Kristiania (Oslo) also in 1898 in which he says he had extended the earlier work of Helmholtz and Kelvin and provided the general analytical derivation of his theorem. Until page 14 of the main paper it is assumed that the reader is aware of, and understands, this background (i.e., the circulation theorem). There is extensive discussion of the components, such as pressure and density surfaces and their orientation, that makes up the practical application of the theorem; see Fig. 2 of Bjerknes (1898), reproduced here as Fig. 3; the definition of the mobility vector is given later in this article. Note the similarity to Silberstein's diagram reproduced here as Fig. 1. 

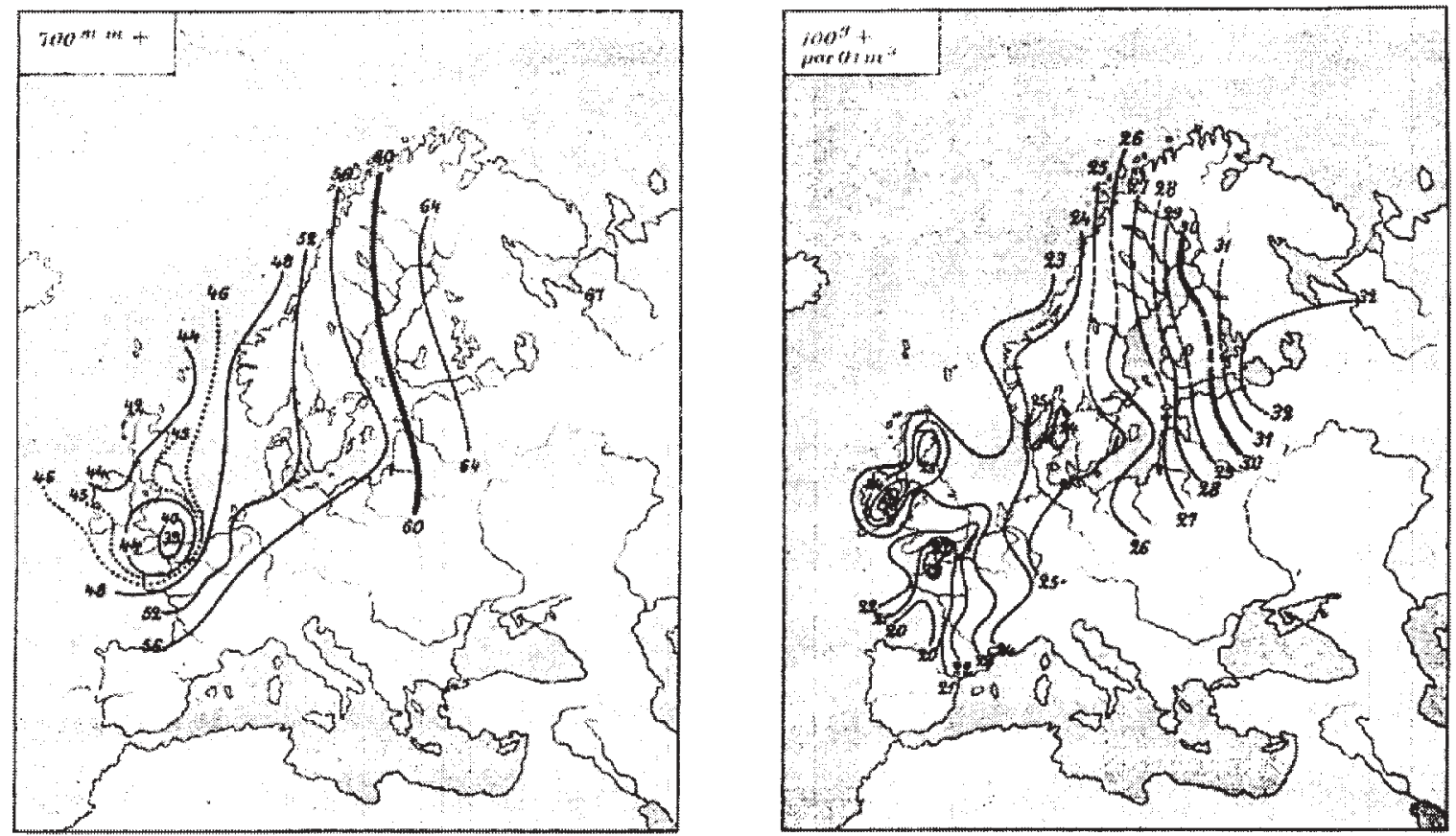

FIG. 2. Reproduction of a figure in Ekholm (189I), which also appears in Kutzbach (1979) as Fig. 32. On the left is pressure and the right density contours for 24 Oct 1882. Tongues of low and high density air are apparent over England within a developing storm.

In the fourth chapter (Rotation and Circulation in a Fluid), the discussion goes back to basics and the paper by Silberstein is referred to (via a reference to the title in French). On page 17 in the section titled "Circulation of fluid curves," Bjerknes states, as theorem III, the circulation theorem:

A closed curve in a fluid possesses, in its circular motion, an acceleration which is equal to the number of the isobaric-isosteric solenoids it encloses.

To modern eyes the mathematics surrounding this statement of the famous theorem is sketchy and unconvincing. The above statement, though, is evocative and makes explicit Bjerknes' background in electromagnetism (the use of the term solenoid). Note that Bjerknes uses the specific volume $k(=1 / \rho)$, in his notation, rather than density itself and hence the reference to isosteres.

It helps at this point in the discussion to digress and describe the circulation theorem mathematically with

modern terminology. In a frictionless flow the (relative) circulation changes according to the following equation:

$$
\frac{D C}{D t}=-\oint k d p,
$$

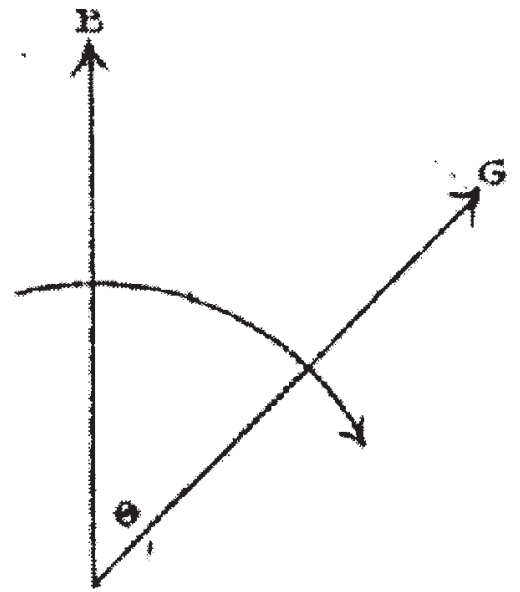

Fig. 3. Reproduction of Fig. 2 from Bjerknes (1898), shows the sense of the developing rotation from the mobility vector $B$ toward the pressure gradient vector $\mathbf{G}$. where $p$ is air pressure.

This arises by differentiating the circulation, following a fluid parcel, that is,

$$
\frac{D C}{D t}=\frac{D}{D t} \oint \mathbf{u} \cdot d \mathbf{l},
$$

which leads to the Bjerknes theorem, (5), if the equations of motion are used to replace the acceleration in terms of the pressure gradient force per unit mass and the geopotential gradient. The latter term disappears for a line integral around a circuit, leaving the term in pressure and specific volume; see (5).
Equation (5) can also be written in the following form, using Stokes' theorem: 


$$
\frac{D C}{D t}=\iint \mathbf{B} \times \mathbf{G} \cdot d \mathbf{A}
$$

where $\mathbf{B}=\nabla k$ and $\mathbf{G}=-\nabla p$. If surfaces of constant $k$ and $p$ coincide, as they do in a homogeneous fluid, then the circulation is constant (Kelvin's theorem).

Eliassen and Kleinschmidt (1957) call $\mathbf{B} \times \mathbf{G}$ the baroclinicity vector. Here we have used Bjerknes' original notation for $\mathbf{B}$ the "mobility" vector and $\mathbf{G}$ the "pressure gradient" vector. (Bjerknes' terminology in quotation marks.) Bjerknes makes a point of defining $\mathbf{B}$ with a positive sign, whereas $\mathbf{G}$, with its minus sign, points in the same direction as the pressure gradient force, from high to low values of pressure. The mobility vector points from low to high specific volume or, if we temporarily take pressure to be constant, from low to high temperature (using the perfect gas law). A possible interpretation for this, and the name mobility, is that molecules are more mobile if temperature is higher. In any event, (7) shows that circulation develops in the direction moving from $\mathbf{B}$ to G; see Fig. 2 of Bjerknes (1898), reproduced here as Fig. 3. Whether the fact that the angle between $\mathbf{B}$ and $\mathbf{G}$ leads to the development of circulation implies a dual meaning of the term mobility is unclear. The different signs used in the definitions of $\mathbf{B}$ and $\mathbf{G}$ seemed to intrigue Bjerknes, who devotes more space in his various papers on the theorem to this aspect than its intrinsic importance might suggest is strictly necessary.

The scene is now set for the various practical applications of the circulation theorem that occupy the remainder of the 1898 paper and subsequent papers (Bjerknes 1900, 1902) on the subject.

\section{APPLICATIONS OF THE BJERKNES' THEOREM. In} considering the practical application of the theorem, Bjerknes noted that if we plot surfaces of $p$ and $k$, with unit interval, then each parallelogram thus formed contributes to the integral in (5) a value of either +1 or -1 . Then the circulation will change if the number of +1 parallelograms (or solenoids) is different to the number of -1 solenoids along the prescribed circuit. So one

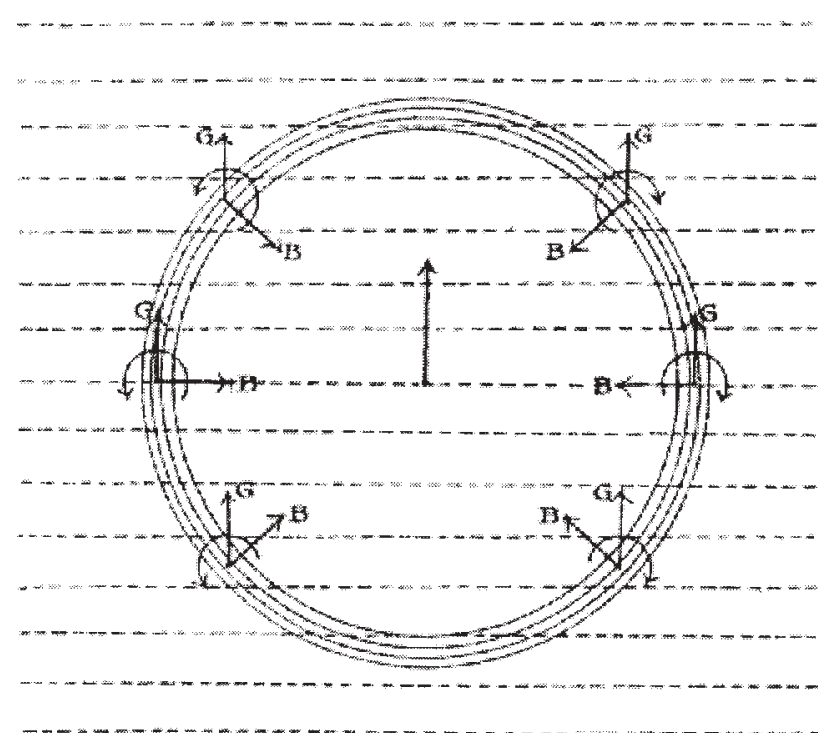

Fig. 4. Reproduction of Fig. 6 from Bjerknes (1898), shows isobaric surfaces with dashed lines (with the pressure gradient vector $\mathbf{G}$ ) and isosteric surfaces as solid lines. This illustrates Archimedes' principle with a mass of less dense air and the consequent development of circulation leading to the ascent of the mass.

has only to count the number of solenoids. His use of the term solenoid comes from his background in electromagnetism and from the associated vector algebra.

In all the examples in the 1898 paper the circulation is considered to be occurring around a circuit that is in the vertical plane. Because pressure decreases upward, $\mathbf{G}$ points predominantly upward in the atmosphere. Bjerknes then considers various examples of distributions of surfaces of specific volume superimposed on the pressure surfaces.

The many applications of the circulation theorem occupy the majority of the 1898 paper. There are sections on production of rotation through heating, Archimedes' principle, air motion in a chimney, trade winds, land and sea breezes, monsoons, cyclones, anticyclones, and circulations in the oceans. We show here some of the figures from the 1898 paper

Fig. 5. Reproduction of Fig. 7 from Bjerknes (1898), which shows the flow of warm air up a chimney with the inflow into the base of the chimney as a consequence of the circulation. 


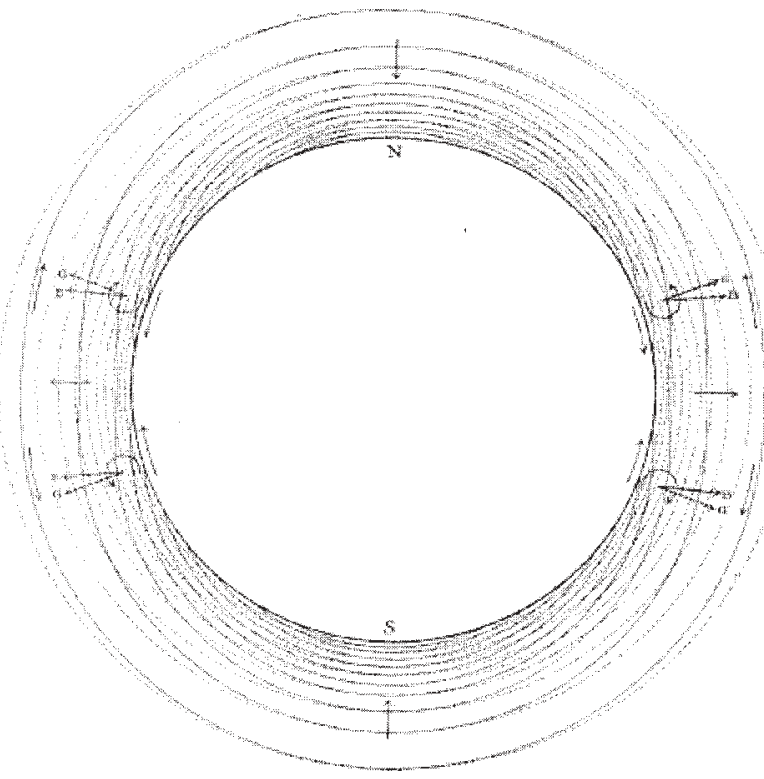

Fig. 6. Reproduction of Fig. 8 from Bjerknes (1898). It shows the global distribution of isobars and isosteres with the consequent meridional circulation. Bjerknes uses this deduction to account for the subtropical trade winds. that relate to these applications. [Figures 6, 7, 8, 9, 10 , and 14 from Bjerknes (1898) are reproduced here as Figs. 4, 5, 6, 7, 8, and 9.] In all of these areas the discussion in the paper is qualitative only and the diagrams are schematics.

Vilhelm Bjerknes also published two papers in Meteorologische Zeitschrift on matters related to the circulation theorem. The first, in 1900, was titled "The Dynamical Principle of Circulatory Motions in the Atmosphere." This paper largely reiterates and summarizes the basic theory in the 1898 treatise, again focusing on the practical calculation of the solenoidal contributions; see Fig. 10, which reproduces Fig. 3 of the 1900 paper. He shows in simple terms how the contribution to the integral in (3) associated with the solenoid in Fig. 10 is $\left(k_{1}-k_{0}\right)\left(p_{1}-p_{0}\right)$. The paper again notes that Silberstein had previously discovered the generalization to Helmholtz's vorticity theory. The paper ends abruptly with the statement "Fortsetzung folgt." or "continuation to follow"!

In 1902 he published a paper titled "Circulation Relative to the Earth" in which a more mathematical, and modern looking, description is given including

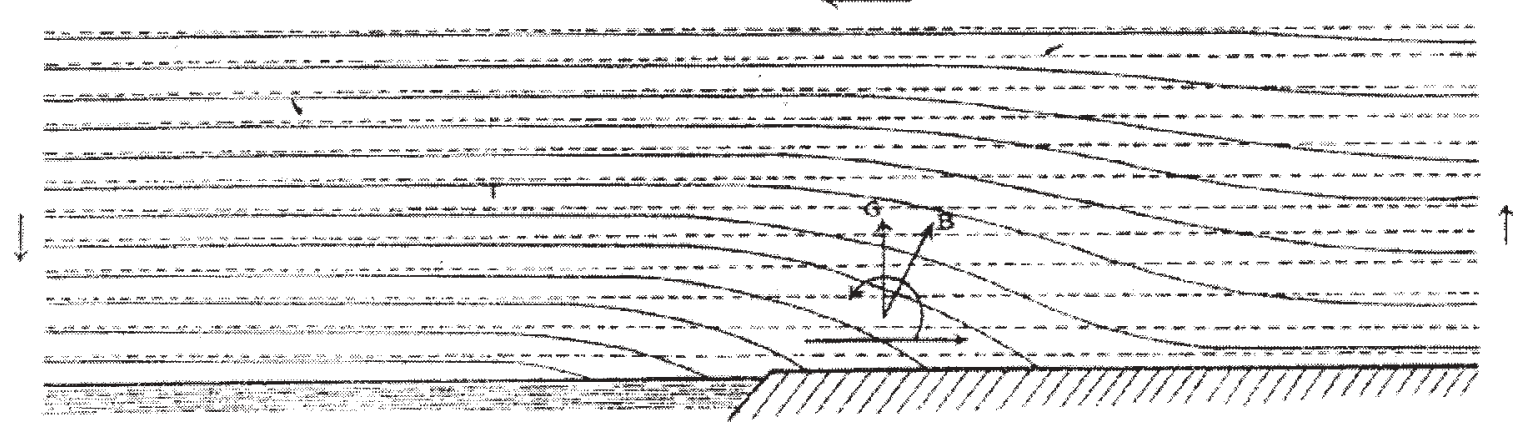

FIG. 7. Reproduction of Fig. 9 from Bjerknes (1898), which shows horizontal isobars across a coastline with less dense air over the land presumably due to solar heating of the ground. The circulation that is thus produced by the baroclinic generation is the sea breeze.

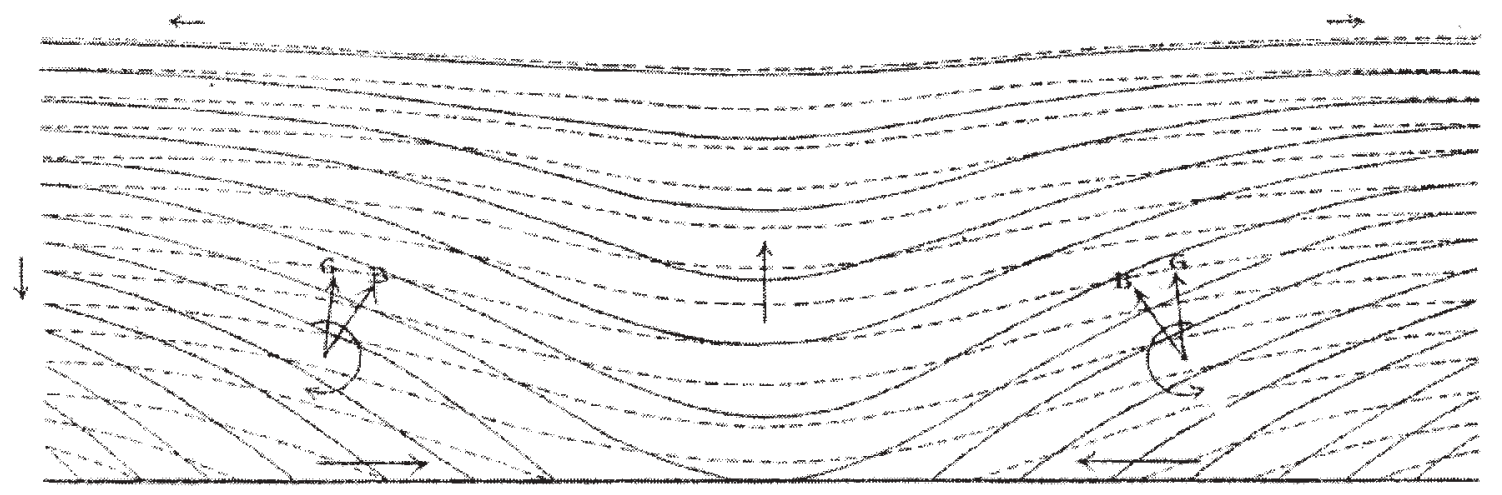

FIG. 8. Reproduction of Fig. I 0 from Bjerknes ( I898), which shows the disposition of isobars and isosteres in an extratropical cyclone, with lower pressure at the central axis with warmer, less dense air there also. The circulation associated with the solenoids produces a direct circulation with warm air rising at the cyclone axis. 
definition and analysis of the absolute and relative circulation contributions. The paper was poorly proofread with missing labels for sections 3 and 5. Bjerknes notes that he gave lectures on the subject to the Stockholm Hochschule in Lent during the 1901 term and had enlisted the help of a student, Johan W. Sandström, to pursue the practical consequences of the new theorem.

A paper by Sandström was also published in $\mathrm{Me}$ -

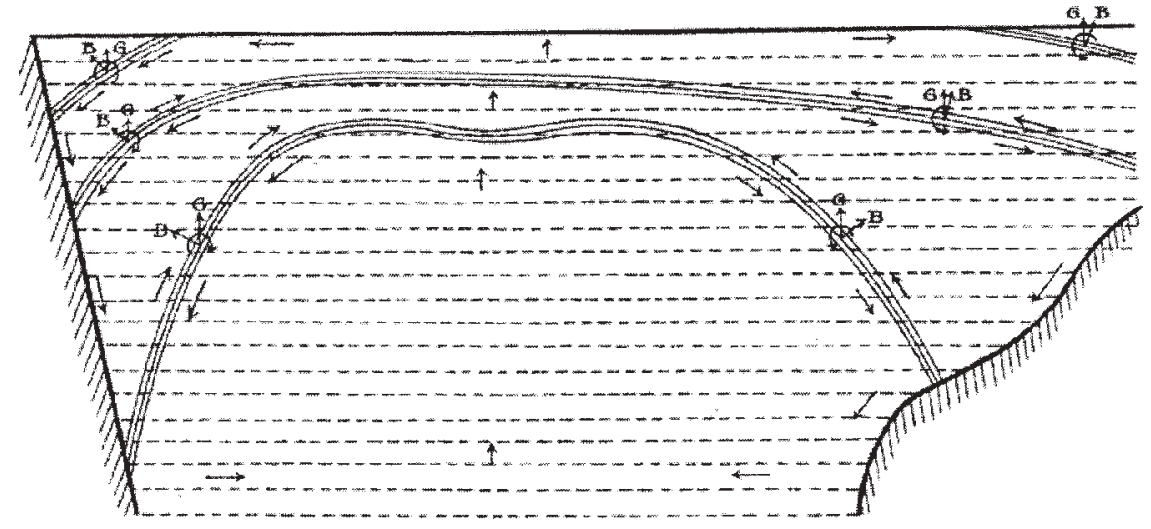

FIG. 9. Reproduction of Fig. 14 from Bjerknes (1898). It shows upwelling and downwelling in the ocean caused by the circulation associated with layers of sharp density gradients. teorologische Zeitschrift

(Sandström 1902) titled "On the Relation Between Temperature and (Air) Motion in the Atmosphere under Stationary Conditions." Ekholm had recommended Sandström to Bjerknes, and for the storm of 21-24 September 1898, which was observed by kite measurements from the Blue Hill Observatory near Harvard University, Sandström constructed the isobaric and isosteric surfaces. The steady-state assumption was made and Sandström showed (in an earlier 1900 paper) a cross section through the cyclone; see Fig. 11 [which reproduces Fig. 37 from Kutzbach (1979), taken from Sandström (1900)]. For the contour interval chosen each parallelogram contributed $10^{5}\left(\mathrm{~cm}^{2} \mathrm{~s}^{-2}\right)$ solenoids. To the left of the cyclone central axis there are about $9 \times 10^{6}$ solenoids while to the right there are about $12 \times 10^{6}$ solenoids, consistent with rising motion along the cyclone axis. Kutzbach notes that Sandström was a former millhand who came to Stockholm on a stipend from his factory. Bjerknes later said that "I would not have concerned myself any further with the alien sciences of meteorology and oceanography had not... my student Sandström been prepared to undertake related practical work in this direction...." (Kutzbach 1979).

Fridtjor Nansen, the renowned Arctic explorer and statesman, read Bjerknes' 1898 paper and wrote to him to ask if the circulation theorem could be applied to the "dead water" phenomenon (Friedman 1989). This had caused problems to Nansen during his voyages on board the Fram in the Arctic. Bjerknes asked another of the students attending his lectures, V. Walfrid Ekman, to look into the problem. In the following year Nansen asked Bjerknes about why his ship and floating ice drifted at a significant angle to the wind. Apparently Ekman was also given this prob- lem, as an application of the circulation theorem, and over the course of one evening derived what is now called the Ekman spiral (Friedman 1989).

SPECIAL CIRCUITS. In the papers Bjerknes published, there appears to be relatively little attention given to the choice of the line (curve) along which the rate of change of circulation is calculated. In nearly all cases this line is implicitly taken to be in the vertical plane. Clearly such circuits will be deformed in time as air parcels that make up the circuit move relative to one another. However one can consider special circuits around which the circulation is conserved. An obvious (but uninteresting) one is if the circuit lies

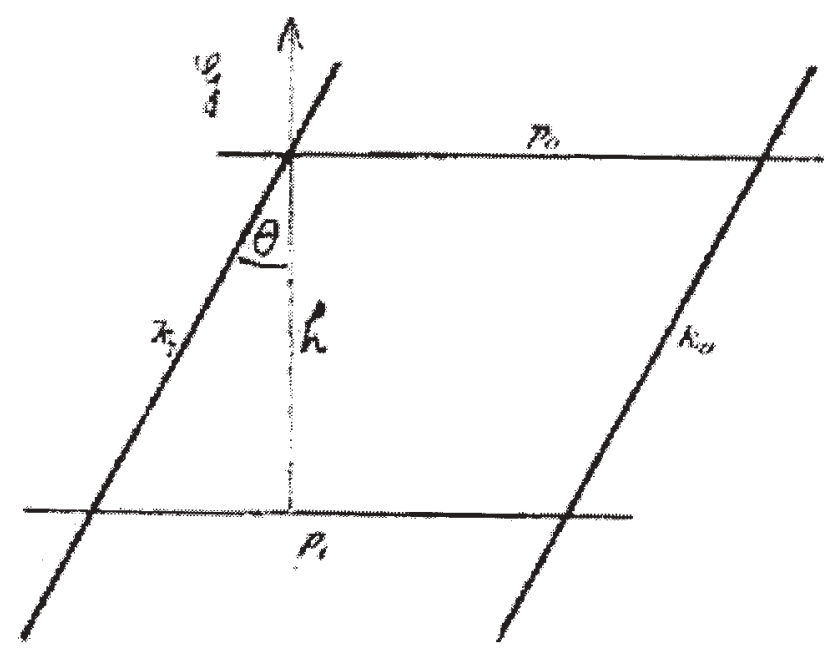

FIG. I0. Reproduction of Fig. 3 from Bjerknes (I900). It demonstrates the elementary parallelogram (solenoid) between intersecting isobars and isoteres. Such a solenoid leads to a contribution to the rate of change of circulation of $\Delta k \Delta p$, which for the unit contour interval is equal to unity. 
on an isobaric surface, in which case the line integral in (5) is instantaneously identically zero.

An interesting choice involves a material circuit that lies on an isentropic surface in the case that the potential temperature, $\theta$, is conserved. Clearly in this case the circuit will lie on that isentropic surface for all time. One can rewrite (5) in the following way, using the perfect gas law and the definition of potential temperature:

$$
\frac{D C}{D t}=-\oint \theta d \pi
$$

where $\pi$ is the Exner function $\left[\pi=c_{p}\left(p / p_{o}\right)^{R / c_{p}}\right.$, with gas constant $\mathrm{R}$, specific heat of air at constant pressure $c_{p}$, and $p_{0}=1000 \mathrm{mb}$.

Clearly circulation is conserved for a circuit that has the property of uniformity in $\theta$. This realization adds a new dimension to the circulation theorem and paves the way for the link to the concept of potential vorticity. Rossby (1940) showed that the absolute circulation per unit mass is a constant for adiabatic frictionless flow and thereby defined a quantity he called the potential vorticity.

THE RELATIONSHIP TO THE VORTICITY EQUATION. We have already noted that Schütz and Silberstein deduced the related result in the context of the vorticity evolution equation, first explored by Helmholtz. Here we make that connection explicit. The full vorticity equation for a frictionless flow can be written as

$$
\begin{aligned}
& \frac{D}{D t} \zeta=(\zeta \cdot \nabla) \mathbf{u} \\
&-\zeta(\nabla \cdot \mathbf{u})+k \mathbf{B} \times \mathbf{G},
\end{aligned}
$$

and the vorticity can be changed by (first term) stretching, tilting, or twisting of existing vorticity; by (second term) fluid expansion or contraction; and by (third term) the baroclinic term. We can see that the baroclinicity vector also appears on the right-hand side of the Bjerknes circulation theorem, (7). The second term in the vorticity equation (9) contributes to changing the absolute circulation [it is the relative circulation that is given in (7)] while the first term in (9) can only redistribute vorticity. Hence the circulation approach, traceable back to Kelvin, and the vorticity approach, traceable back to Helmholtz, are entirely consistent with one another.

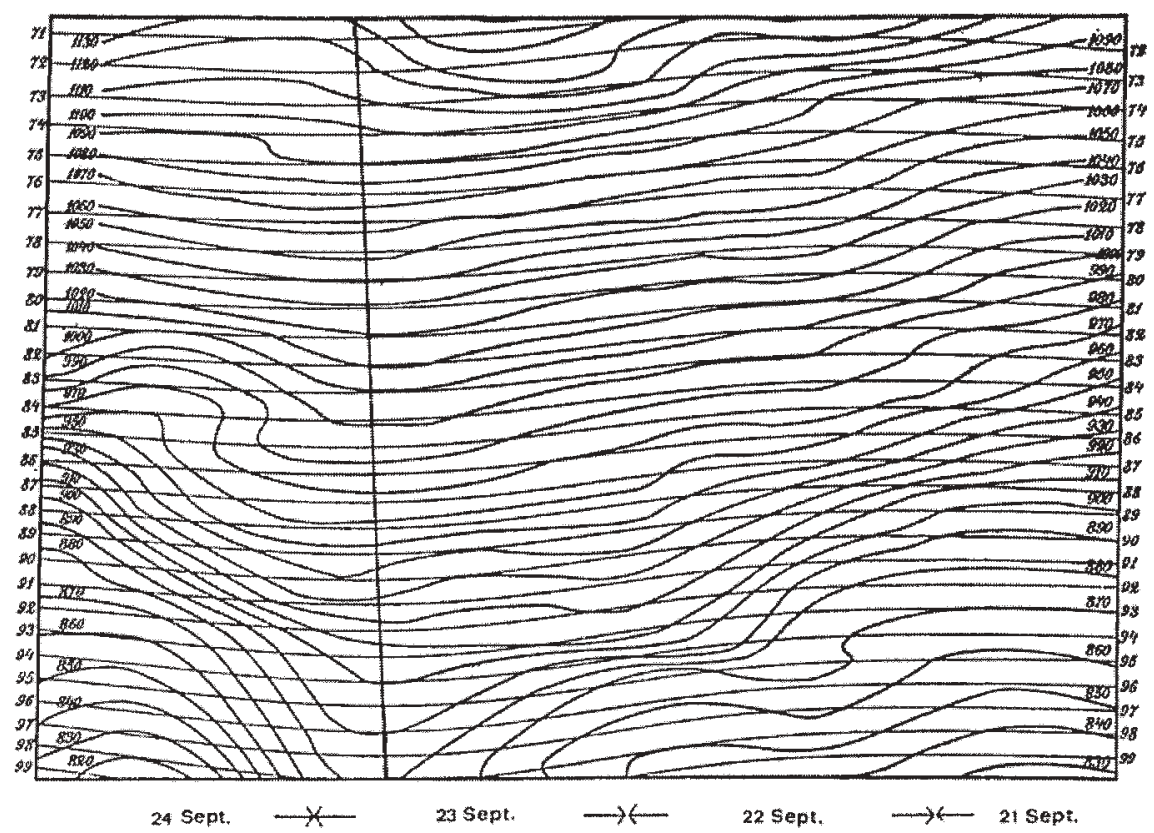

Fig. II. Reproduction of a figure in Sandström (1900), and shown in Kutzbach (1979) as Fig. 37. It shows a vertical cross section of isobars and isosteres for a storm over the Blue Hill Observatory, Milton, MA, on 2I-24 Sep 1898. Isobars are given with contour intervals of $10^{4} \mathrm{~g} \mathrm{~cm}^{-1} \mathrm{~s}^{-2}$ and isoteres with contour intervals of $10 \mathrm{~cm}^{3} \mathrm{~g}^{-1}$. In this diagram, each parallelogram contributes $10^{5}$ solenoids to the rate of change of circulation. The vertical axis, added by Kutzbach, indicates the central axis of the cyclone.
DISCUSSION. It has been shown how the Bjerknes' circulation theorem extended the earlier Kelvin circulation theorem. In parallel Schütz and Silberstein extended the earlier Helmholtz derivation of the vorticity evolution equation for nonhomogeneous fluids. Schütz did not provide any physical interpretation of the new vorticity equations whereas Silberstein provided such an insight. The explicit link to geophysical fluids was not made until Bjerknes' work. The circulation and vorticity approaches are consistent with one another and lead naturally onto the conservation of potential vorticity for "ideal" fluids. The meteorological insight given by the 
Bjerknes' theorem is considerable although it is not now common practice to routinely diagnose the circulation or its rate of change.

A key feature of the Bjerknes theorem is that it signaled the move of Vilhelm Bjerknes into meteorology. He was an ambitious scientist and the Bergen School, which he established 20 years after the publication of his theorem, introduced many concepts about extratropical cyclones and weather forecasting that have had lasting value. Even as early as 1904 Vilhelm Bjerknes set the agenda for the subsequent development of numerical weather forecasting in his seminal paper, Bjerknes (1904). One might argue that no dynamical concept as profound as the circulation theorem emerged during the heyday of the Bergen School but that would be to lose sight of the school's tremendous conceptual impact. Vilhelm Bjerknes certainly had a profound interest and understanding of the development process of various subdisciplines of physics such as mechanics, thermodynamics, hydrodynamics, and meteorology. He placed the theoretical and applied contributions by himself and his various research groups into this perspective [cf. his personal reflections in Bjerknes (1938)].

While circulation and its tendency are not commonly calculated for weather systems, the legacy of that approach perhaps needs to be heeded more often than is currently the case. We know that point estimations of vorticity and potential vorticity suffer from having an ultraviolet spectrum-the smaller scale the observations are, the more structure is seen. Area integrals are meaningful however and the message from Bjerknes' circulation theorem is that whether it is called circulation or area-averaged vorticity, it is the quantity that forms a critical building block of dynamical thinking.

ACKNOWLEDGMENTS. This paper arose from a talk given to a Royal Meteorological Society meeting organized by Ian Roulstone, in November 2001. The authors wish to thank Sigbjorn Grønås and Harald Lejanäs, for assistance in obtaining copies of some of the original Bjerknes papers. Anna Sienkiewicz of the Jagiellonian University Library in Cracow gave us substantial advice and help to find the paper by, and historical information about the life of, Ludwik Silberstein, and Bronisław Średniawa helped us find the Schütz paper.

\section{REFERENCES}

Bjerknes, V., 1898: Über einen hydrodynamischen

Fundamentalsatz und seine Anwendung besonders auf die Mechanik der Atmosphäre und des
Weltmeeres. Kongl. Sven. Vetensk. Akad. Handlingar, 31, 1-35.

—, 1900: Das dynamische Princip der Circulationsbewegungen in der Atmosphäre. Meteor. Z., 17, 97-106. — 1902: Cirkulation relativ zu der Erde. Meteor. Z., 19, 97-108.

__, 1904: Das Problem der Wettervorhersage, betrachtet vom Standpunkte der Mechanik und der Physik (The problem of weather forecasting as a problem in mechanics and physics). Meteor. Z., 21, 1-7. (English translation by Y. Mintz, 1954, reproduced in The Life Cycles of Extratropical Cyclones, 1999, Amer. Meteor. Soc.)

_-, 1938: Leipzig-Bergen. Festvortrag zur 25-Jahrfeier des Geophysikalischen Instituts der Universität Leipzig. Z. Geophys., 14, 49-62. (English translation by L. Shields, 1997, jubilee address of the 25th anniversary of the Geophysical Institute of the University of Leipzig, Historical Note 2, Irish Meteorological Service, Dublin, Ireland, 17 pp.)

Ekholm, N., 1891: Étude des conditions météorologiques a l'aide de cartes synoptiques représentant la densité de l'air. Kongl. Sven. Vetensk. Akad. Handlingar, 16, 14-15.

Eliassen, A., and E. Kleinschmidt, 1957: Dynamic meteorology. Handbuch der Physik, S. Flügge, Ed., Vol. 48, 1-154.

Friedman, R. M., 1989: Appropriating the WeatherVilhelm Bjerknes and the Construction of a Modern Meteorology. Cornell University Press, $251 \mathrm{pp}$.

Gill, A. E., 1982: Atmosphere-Ocean Dynamics. Academic Press, 662 pp.

Helmholtz, H., 1858: Über Integrale der hydrodynamischen Gleichungen, welche den Wirbelbewegungen entsprechen. J. Angew. Math., 55, 25-55.

Kutzbach, G., 1979: The Thermal Theory of Cyclones: A History of Meteorological Thought in the Nineteenth Century. Amer. Meteor. Soc., 255 pp.

Rossby, C.-G., 1940: Planetary flow patterns in the atmosphere. Quart. J. Roy. Meteor. Soc., 66 (Suppl.), 68-87.

Sandström, J. W., 1900: Über due Anwendung von Professor V. Bjerknes' Theorie der Wirbelbewegungen in Gasen und Flüssigkeiten auf meteorologische Beobachtungen in den höheren Luftschichten. Kongl. Sven. Vetensk. Akad. Handlingar, 33, 23.

__, 1902: Über die Beziehung zwischen Temperatur und Luftbewegung in der Atmosphäre unter stationären Verhältnissen. Meteor. Z., 19, 161-170.

Schütz, J. R., 1895: Über die Herstellung von Wirbelbewegungen in idealen Flüssigkeiten durch conservative Kräfte. Ann. Phys. Chem., 56, 144-147. 
Silberstein, L., 1896: O tworzeniu się wirów w płynie doskonałym. W Krakowie Nakładem Akademii Umiejętności, Skład Główny w Księgarni Spółki Wydawniczej Polskiej, 325-335. [Also published as Über die Entstehung von Wirbelbewegungen in einer reibungslosen Flüssigkeit in Bull. Int. l'Acad.
Sci. Cracovie, Compt. Rend. Séances Année, 1896, 280-290 (but without its figures)].

Średniawa, B., 1997: Ludwik Silberstein. Polski Słownik Biograficzny (Polish Biographical Dictionary), Polish Academy of Sciences, 482-483.

Thomson, W., 1867: On vortex atoms. Proc. Roy. Soc. Edinburgh, 6, 94-105. 